\title{
Entrelacs
}

Cinéma et audiovisuel

18 | 2021

Laurent Roth, cinéaste

\section{Trois présentations}

\section{Yannick Haenel}

\section{(2) OpenEdition}

Journals

Édition électronique

URL : https://journals.openedition.org/entrelacs/6092

DOI : 10.4000/entrelacs.6092

ISSN : 2261-5482

Éditeur

Éditions Téraèdre

Référence électronique

Yannick Haenel, «Trois présentations », Entrelacs [En ligne], 18| 2021, mis en ligne le 14 décembre

2021, consulté le 15 décembre 2021. URL : http://journals.openedition.org/entrelacs/6092 ; DOI :

https://doi.org/10.4000/entrelacs.6092

Ce document a été généré automatiquement le 15 décembre 2021.

Tous droits réservés 


\title{
Trois présentations
}

\author{
Yannick Haenel
}

\section{I - Parole de Yannick Haenel sur les films du Jardin}

\author{
(Entre Marie ou le retour et Ave Maria suivi de Modèle depuis toujours) \\ «Vous avez vu ce tout premier film de Laurent Roth \\ où il y a un enfant, \\ un enfant qui est un fils, \\ - c'est Pasolini qui disait que tout cinéma est cinéma « du fils» -, \\ Vous avez vu cette épée que l'on essaye de sortir d'un jardin, \\ Vous avez vu cette femme qui est a la fois une mère adorée \\ et une amante possible, à venir, \\ comme dans Phèdre... \\ Il se trouve que, \\ quand, ayant la joie de découvrir tous les petits films, \\ - ce n'est pas péjoratif " petits films ", c'est comme pour un poème - \\ tous les petits films de Laurent Roth, \\ je me suis aperçu qu'au début, \\ tout se passait dans un jardin, \\ même si on regarde ce jardin depuis une maison \\ que l'on habite pas vraiment, \\ et qu'après, en grandissant, ce cinéma et Laurent lui même, \\ passe dans, \\ ou essaye d'habiter une maison. \\ Je me suis posé une question, \\ c'est celle que posent les deux prochains petits films que vous allez voir \\ qui est celle de l'innocence \\ tout simplement, \\ je dis « tout simplement ", mais en italique, \\ car je crois que c'est la grande question du cinéma, \\ en tous les cas de la littérature, \\ de ce qu'on peut aimer dans les deux, \\ celle de savoir si le fait de passer du jardin à la maison \\ est une rupture d'innocence, \\ ou bien s'il est possible de retrouver quelque chose, \\ le temps,
}


l'amour,

et tout ce que vous savez...

Voilà,

j'aime bien l'expression :

«bonne projection",

on en rigolait tout à l'heure avec Dominique Cabrera,

" projetez vous bien ",

vous allez voir

ça y va!»

\section{II - Parole de Yannick Haenel sur les films de la Famille}

(Entre Ranger les Photos et Une maison de famille)

"J'aime ce moment de Ranger les Photos

Lorsque Dominique Cabrera retrouve une photo d'elle

Une photo d'elle enfant

ce moment où elle dit : « je me demande ce que je pense ",

je crois que c'est mieux que Heidegger en fait,

c'est un film qui donne infiniment à penser

penser le temps,

l'identité de la pensée et du temps,

et de manière gracieuse,

et puis...

Je n'avais jamais pensé à ça,

mais puisque Vincent Dieutre est présent ce soir,

j'associe avec Fragments sur la grâce

au fait que la grâce peut être incarnée par un moment

où on enlève le vernis bleu de ses ongles,

comme à la fin du film

c'était juste pour dire ça

Voilà

Vous avez vu des films qui se passent dans le jardin

Et voici maintenant un film central me semble-t-il de Laurent,

un petit peu plus long,

ce n'est pas qu'il se passe dans une maison,

c'est qu'il cherche à retrouver la maison.

Moi quand j'étais petit,

je ne sais pas vous

mais mes parents n'arrêtaient pas de me dire :

« Tiens toi bien!»

et j'imagine que c'est parce que j'étais un peu voûté,

je ne sais pas,

mais cette phrase m'est restée

et c'est pas que j'essaye de bien me tenir

peut-être même c'est le contraire,

mais je crois que c'est l'un des sujets de ce film,

un peu fou, oui, de Laurent,

où il s'agit de se demander me semble-t-il,

comment est-ce qu'on se tient vis a vis de sa famille,

comment est-ce que sa famille,

notre propre famille,

imagine qu'on "se tient ",

est-ce qu'il est possible de se tenir déjà,

ou « de se tenir de soi-même "

comme dit Maître Eckhart?

Ce qui est frappant, 
vous allez voir,

je ne vais pas trop en dire,

c'est que, eh bien, on voit Laurent,

qui assume la position du narrateur,

moi ça me touche,

parce qu'il y va carrément,

enfin c'est pas simplement pour faire de l'autobiographie

mais c'est sans doute parce qu' il y a un moment où,

quand on fait des films,

quand on écrit un livre,

il faut passer à l'acte,

il ne faut plus seulement s'y croire ou simuler

pour toucher au cinéma

mais se laisser être pour que le cinéma advienne,

pour que des phrases s'écrivent.

Le corps de Laurent dans ce film est un corps burlesque :

Il y a des pages très belles de Jean-Louis Schefer,

je ne sais pas si vous aimez cet écrivain

qui parle très bien de la peinture

mais aussi du cinéma

et, je ne sais plus trop où,

j'ai lu ça il y a trente ans,

il parlait de Buster Keaton

il dit que le visage grave et soit disant figé de Buster Keaton

est en fait

une demande d'amour.

Ainsi vous allez voir comment Laurent passe,

en faisant le fou,

puisqu'il il ne sait pas " comment se tenir ",

enfin ce narrateur qui s'appelle Laurent Roth,

en faisant le fou car il ne sait pas comment « se tenir "

devant sa famille,

va être obligé de devenir

chef de chœur,

ou chef d'orchestre

ou cinéaste

Bonne projection. »

\title{
III - Parole de Yannick Haenel sur les films de la Parole
}

\author{
(Avant Le Pays fantôme) \\ "Le cinéma de Laurent évoque souvent des lieux \\ dans ses titres, \\ et là vous allez visiter un Pays, \\ même s'il est fantôme, \\ vous allez voir, \\ j'en dis juste deux mots, \\ je crois me souvenir que Laurent a trouvé une bobine, \\ il a acheté une bobine ancienne, \\ et à partir de cette bobine, \\ qui se passe dans une caserne, \\ qui est un lieu presque initiatique pour lui, \\ dans son œuvre et dans sa vie puisqu'il a commencé... \\ Il a non pas commencé à filmer, \\ mais il a fait du cinéma aussi grâce à l'armée \\ et à cette histoire du cinéma et de l'armée...
}


Et donc,

à partir de cette bobine, muette,

le film va offrir,

ce que l'on appelait dans les années 70 :

"l'engendrement de la formule",

il va par la parole essayer de trouver une fiction

ou des fictions possibles,

déployer des vies,

déployer des voix de ces quelques minutes,

c'est-à-dire de ressusciter pour le dire de manière chrétienne et juive,

ressusciter quelque chose qui a été enregistré,

mais dont on ne sait pas si ça appartient

à la vie

ou à la mort.

Quel enchantement possible par la fiction,

je crois que Le Pays fantôme c'est ça :

c'est peut-être la fiction!

A vous de voir...»

\section{RÉSUMÉS}

À l'occasion de l'édition DVD de l'intégrale de ses courts-métrages (La Huit), Laurent Roth invite Yannick Haenel à participer à une projection-performance le mardi 25 février 2020 à Paris au cinéma l'Archipel. Nous retranscrivons ici les trois présentations de Yannick Haenel qui se sont articulées autour du programme suivant: I. Films du Jardin: Marie ou le retour (1984, 10'), Ave Maria $\left(1987,4^{\prime}\right)$ et Modèle depuis toujours $\left(1988,10^{\prime}\right)$; II. Films de la Famille : Ranger les Photos (1998, 14 ', co-réal. D. Cabrera, L. Roth), Une maison de famille $(2004,34$ ') ; III. Films de la Parole : Le Pays fantôme (2019, 10', avant-première).

On the occasion of the DVD edition of his complete short films (La Huit), Laurent Roth invites Yannick Haenel to participate in a screening-performance on Tuesday 25 February 2020 in Paris at the Archipel cinema. We transcribe here the three presentations of Yannick Haenel which were articulated around the following programme: I. Films du Jardin; II. Films of the Family; III. Films of the Word.

\section{AUTEUR}

\section{YANNICK HAENEL}

Écrivain et essayiste, il a passé sa jeunesse au Prytanée Militaire de la Flèche (séjour qu'il a relaté dans son premier roman : Les Petits Soldats, paru en 1996 aux éditions de la Table Ronde). Il a reçu le prix Interallié et le Prix du roman Fnac pour Jan Karski (Gallimard, 2009). En 2017, il publie le roman Tiens ferme ta couronne, finaliste du Prix Goncourt, et lauréat du Prix Médicis. Yannick Haenel est chroniqueur pour le magazine de littérature et de cinéma Transfuge, depuis 2010, et à Charlie Hebdo, depuis la reprise de la publication après les attentats de janvier 2015. 